\title{
De Garengeot hernia: Revisited
}

\author{
Rosley M*, Gu J, Russell A, Wullschleger M and Patel B
}

Acute Care Surgical Unit, Gold Coast University Hospital, Gold Coast, Queensland, Australia

\section{Case Report}

A 66 year old woman presented to the Emergency Department with a 4 day history of a right groin lump, which was becoming increasingly painful. An external ultrasound suggested a fat containing femoral hernia. She had a past medical history of atrial fibrillation and was taking Apixaban.

On examination, a firm, slightly erythematous mass was seen in the right inguinal region. It was located inferior to the inguinal ligament, and was tender and irreducible on palpation. The patient was haemodynamically stable, with no symptoms to suggest bowel obstruction or ischaemia.

A portal venous phase CT scan was performed (Figures 1 and 2) which confirmed a fat-containing right femoral hernia. There was no bowel involvement, but the appendix tip was noted to be just within the hernia sac.

As the patient was stable and anticoagulated, she was taken to theatre the following day. Initially, a laparoscopic approach was used to visualise the entire abdominal cavity. There was no gross contamination or bowel involvement, however, the appendix was indeed seen to enter the femoral canal (Figure 3). On reduction of the tip from the hernial sac, it was found to be necrotic (Figure 4) and an appendicectomy was performed. Following this, an open femoral hernia repair via infrainguinal approach was performed. The hernial sac was identified and

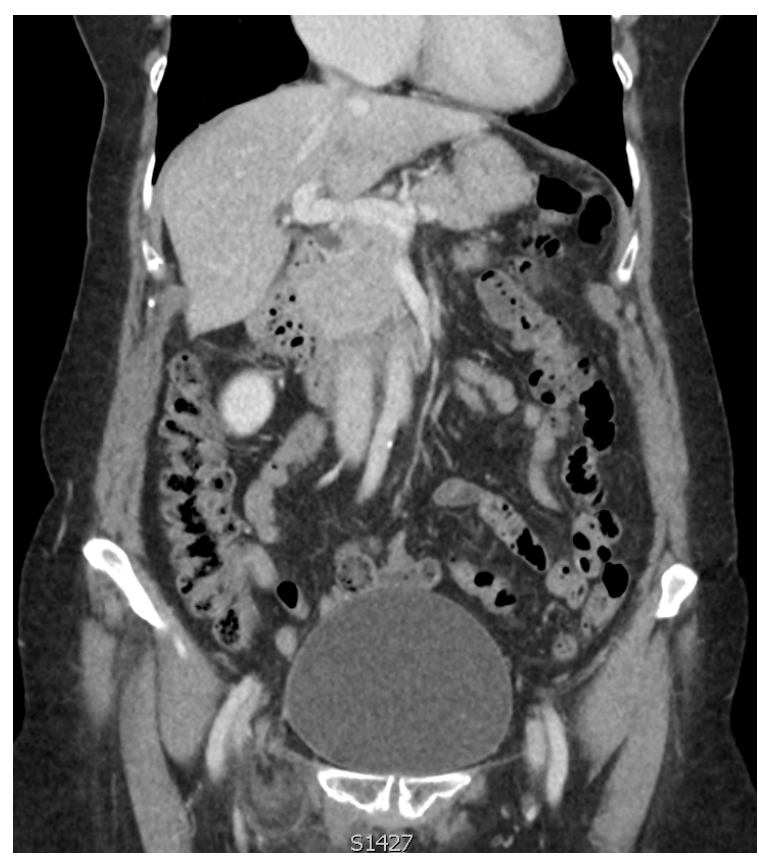

Figure 1. CT scan suggestive viable bowel but? involvement of appendix

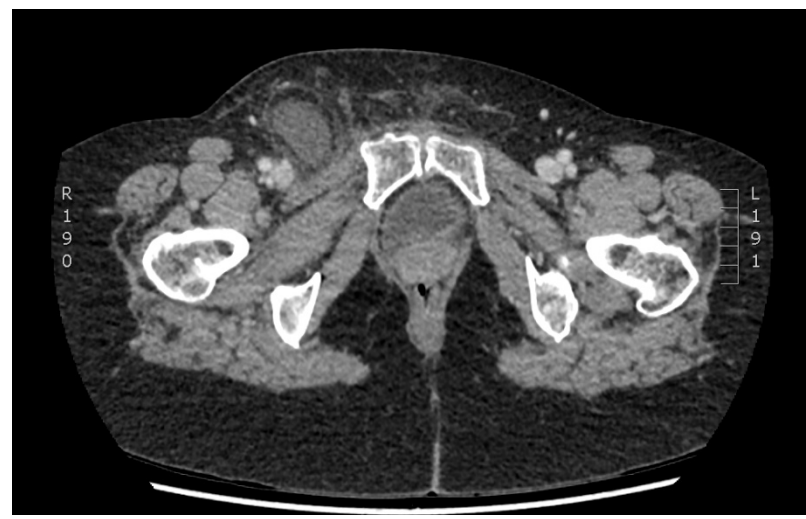

Figure 2. CT scan suggestive of Omentum in the Femoral hernia sac

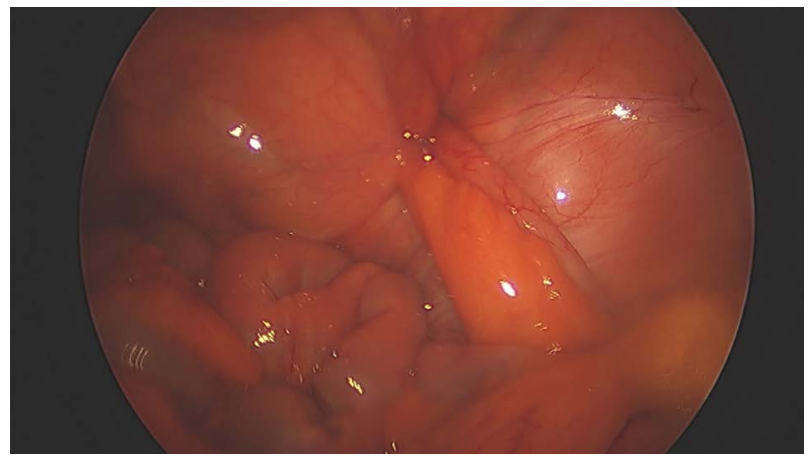

Figure 3. Intra- Operative view of appendix in Femoral canal

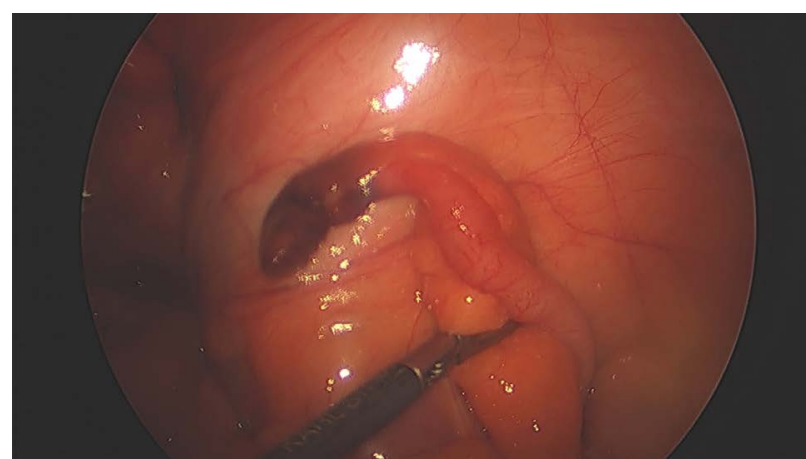

Figure 4. Intra-Operative view of gangrenous tip of the appendix

${ }^{\star}$ Correspondence to: Muhammad Farqhan Rosley, MB BCh BAO, Acute Surgical Unit GCUH, Tel: 0424329525; E-mail: muhammadrosley@rcsi.ie

Received: January 02, 2019; Accepted: January 08, 2019; Published: January 11, 2019 
opened to show still viable omentum, and this along with the sac was suture ligated at the defect and the hernia reduced. Primary repair was carried out by approximating the upturned part of the inguinal ligament to the pectineal fascia using interrupted tension free nonabsorbable suture material. There were no perioperative complications, and the patient was discharged 24 hours later.

Histological study of the appendix showed vascular congestion and fat necrosis in the peri-appendicular fatty tissue with no evidence of neoplasia.

\section{Discussion}

This rare hernia was first described by Rene Jacques Croissant de Garengeot, a French surgeon in 1731 [1]. The first appendicectomy in de Garengeot hernia was performed by Hevin many years later in 1785 [2].

The rare incidence of this condition allows no consensus on the best surgical approach. Being on apixaban, we concluded that the chosen approach will give us the best haemostatic control and reduce possible bleeding issues. This approach has been described previously however open repair with appendicectomy appears to be the preferred method [3-5].

We suggest that this could be a viable approach in cases where preoperative diagnosis of de Garangeot hernia has been made.

\section{References}

1. De Garengeot RJC (2009) Traite des operations de chuirgie. (2nd Edn), Paris: Huart 1731: $369-371$.

2. Akopian G, Alexander M (2005) De Garengeot hernia: appendicitis within a femoral hernia. Am Surg 71: 526-527. [Crossref]

3. Kalles V, Mekras A, Mekras D, Papapanagiotou I, Al-Harethee W, et al. (2013) De Garengeot's hernia: a comprehensive review. Hernia 17: 177-182. [Crossref]

4. Shiihara M, Kato T, Kaneko Y, Yoshitoshi K, Ota T (2017) de Garengeot hernia with appendicitis treated by two-way-approach surgery: a case report. Journal of Surgical Case Reports Issue 7: 1.

5. Thomas B, Thomas M, McVay B, Chivate J (2009) De Garengeot hernia. JSLS 13 455-457. [Crossref]

Copyright: (C2019 Rosley M. This is an open-access article distributed under the terms of the Creative Commons Attribution License, which permits unrestricted use, distribution, and reproduction in any medium, provided the original author and source are credited. 